\title{
Consulta de enfermagem no pré-natal: atendimento à gestante com sífilis
}

\author{
Prenatal nursing consultation: care for pregnant women with syphilis \\ Consulta de enfermería prenatal: atención a mujeres embarazadas con sífilis
}

Recebido: 13/12/2021 | Revisado: 20/12/2021 | Aceito: 25/12/2021 | Publicado: 05/01/2022

\author{
Aline Luiza Deliberalli \\ ORCID: https://orcid.org/0000-0002-0721-0129 \\ Centro Universitário de Pato Branco, Brasil \\ E-mail: alinedeliberalli@gmail.com \\ Vanessa Aparecida Pawnoski \\ ORCID: https://orcid.org/0000-0003-0386-6486 \\ Centro Universitário de Pato Branco, Brasil \\ E-mail: vanepawnoski@hotmail.com \\ Gisele Iopp Massafera \\ ORCID: https://orcid.org/0000-0002-8116-0051 \\ Centro Universitário de Pato Branco, Brasil \\ E-mail: gisele.iopp.massafera.gmail.com \\ Juliane Pagliari Araujo \\ ORCID https://orcid.org/0000-0001-7821-6731 \\ Instituto Federal do Paraná, Brasil \\ E-mail: juliane.pagliari@ifpr.edu.br \\ Lujácia Felipes Fiorentin \\ ORCID: https://orcid.org/0000-0001-6635-7684 \\ Centro Universitário de Pato Branco, Brasil \\ E-mail: lujacia.fiorentin@unidep.edu.br
}

\begin{abstract}
Resumo
Objetivo: Identificar as ações do enfermeiro durante a consulta pré-natal às gestantes com sífilis. Metodologia: Tratase de uma pesquisa exploratória, descritiva com abordagem qualitativa, realizada com oito enfermeiros que realizam assistência pré-natal em um município na região Sudoeste do Estado do Paraná. A coleta de dados ocorreu em maio de 2021 e a entrevista foi gravada e transcrita posteriormente. Os dados foram analisados a partir da técnica de análise temática. Resultados e Discussão: Os enfermeiros realizam consulta de enfermagem durante o pré-natal e atentam aos protocolos de detecção e tratamento precoce da sífilis na gestação. As gestantes são orientadas sobre a doença, bem como, da necessidade precoce do seu tratamento e do seu parceiro para evitar a transmissão vertical e possíveis problemas com recém-nascido. Conclusão: É necessário dar ênfase na busca ativa das gestantes para um início precoce do pré-natal para evitar agravos. As práticas assistenciais podem refletir na qualidade da assistência e indicadores de saúde.
\end{abstract}

Palavras-chave: Atenção primária à saúde; Cuidado pré-natal; Cuidados de enfermagem; Sífilis.

\begin{abstract}
Objective: To identify the actions of nurses during prenatal consultations for pregnant women with syphilis Methodology: This is an exploratory, descriptive research with a qualitative approach, carried out with eight nurses who provide prenatal care in a city in the Southwest region of the State of Paraná. Data collection took place in May 2021 and the interview was recorded and transcribed later. Data were analyzed using the thematic analysis technique. Results and Discussion: Nurses perform nursing consultations during prenatal care and pay attention to protocols for early detection and treatment of syphilis in pregnancy. Pregnant women are advised about the disease, as well as the need for early treatment of their partner and their partner to avoid vertical transmission and possible problems with the newborn. Conclusion: It is necessary to emphasize the active search of pregnant women for an early start of prenatal care to avoid harm. Care practices can reflect on the quality of care and health indicators.
\end{abstract}

Keywords: Primary health care; Prenatal care; Nursing care; Syphilis.

\section{Resumen}

Objetivo: Identificar las acciones de las enfermeras durante las consultas prenatales de gestantes con sífilis. Metodología: Se trata de una investigación exploratoria, descriptiva con enfoque cualitativo, realizada con ocho enfermeras que brindan atención prenatal en una ciudad de la región suroeste del estado de Paraná. La recolección de datos tuvo lugar en mayo de 2021 y la entrevista fue grabada y transcrita posteriormente. Los datos se analizaron mediante la técnica de análisis temático. Resultados y Discusión: Las enfermeras realizan consultas de enfermería durante la atención prenatal y prestan atención a los protocolos de detección precoz y tratamiento de la sífilis en el 
embarazo. A las mujeres embarazadas se les advierte sobre la enfermedad, así como la necesidad de un tratamiento temprano de su pareja y su pareja para evitar la transmisión vertical y posibles problemas con el recién nacido. Conclusión: Es necesario enfatizar la búsqueda activa de gestantes para un inicio temprano de la atención prenatal para evitar daños. Las prácticas de atención pueden reflejar la calidad de la atención y los indicadores de salud.

Palabras clave: Atención primaria de salud; Cuidado prenatal; Cuidado de enfermera; Sífilis.

\section{Introdução}

A sífilis é uma doença infectocontagiosa sistêmica, causada pela bactéria Treponema pallidum, sua principal forma de transmissão é por relação sexual sem preservativo com indivíduos infectados, ou por transmissão vertical, resultando em sífilis congênita e seus agravos aos neonatos. Apesar de o tratamento ser eficaz e de baixo custo, ainda é um grave problema de saúde pública no Brasil (Brasil, 2019c).

Para o diagnóstico da sífilis é necessário inicialmente a realização de uma avaliação clínica que inclua a anamnese e exame físico capaz de identificar a presença dos sinais e sintomas da doença. O exame mais realizado é o teste não treponêmico: VDRL. Para confirmar a reatividade dos testes não treponêmicos são realizados testes treponêmicos que detectam antígenos específicos do T. pallidum, como FTA-ABS, hemoaglutinação e imunofluorescência (Luppi, Domingues \& Gomes, 2016).

No Brasil, no período de 2005 a 2019, foram notificados no Sistema de Informação de Agravos de Notificação (SINAN), 324.321 casos de sífilis em gestantes. O ano de 2018 apresentou maior incidência, com 62.599 casos, sendo observada uma taxa de detecção de 21,4 casos de sífilis em gestantes para cada 1000 nascidos vivos. O estado do Paraná acumulou 1.240 mil casos de sífilis em gestantes e 414 casos de sífilis congênita no ano de 2019, destes, a maioria das contaminações ocorreram em recém-nascidos com menos de sete dias de vida (Brasil, 2019a). A sífilis congênita quando diagnosticada e tratada prematuramente diminui gradativamente o risco de morte fetal.

Com o objetivo de melhorar os indicadores, o acesso e a qualidade da assistência pré-natal, parto e puerpério a gestante e ao recém-nascido, foi instituído em 2000 pelo Ministério da Saúde, o Programa de Humanização no Pré-natal e Nascimento (PHPN), na perspectiva de reduzir as taxas de morbimortalidade materna e perinatal registradas no país (Santos \& Araujo, 2016). Nesse sentido, a assistência pré-natal de qualidade configura-se como uma ferramenta de prevenção, detecção precoce, tratamento e redução de morbidades, o que pode reduzir a prevalência de sífilis nesse grupo populacional (Suto et al., 2016).

Desde 2011 o governo federal vem implementando a "Rede Cegonha", como forma de complementar o PHPN, com o programa vem à perspectiva de mudança no atendimento à mulher no período gravídico/puerperal por parte dos profissionais, devido ao aumento da oferta de testes rápidos para rastreio de sífilis e HIV (Vírus da Imunodeficiência Humana) nas Unidades de Atenção Básica. A Rede Cegonha assegura à mulher e criança o direito de ter atenção humanizada e de qualidade em todas as etapas da gestação e puerpério e nos demais serviços de saúde do Sistema Único de Saúde (SUS) (Brasil, 2015).

Dessa forma, levantou-se a seguinte questão norteadora: Quais as condutas do enfermeiro na atenção pré-natal às gestantes com sífilis?

Diante ao exposto e, partindo do pressuposto de que um pré-natal de qualidade e uma boa interação entre o enfermeiro e a gestante é capaz de diminuir as consequências da sífilis, objetivou-se identificar as ações do enfermeiro durante a consulta pré-natal às gestantes com sífilis.

\section{Metodologia}

O presente trabalho consiste em um estudo exploratório, transverso, descritivo com abordagem qualitativa realizado com enfermeiros que atuam na atenção primária em saúde, realizando atendimento de pré-natal, em um município no sudoeste do estado do Paraná. 
O cenário de estudo abrangeu uma unidade de saúde central do município conectada à Rede Mãe Patobranquense e cinco Unidades de Estratégias de Saúde da Família, localizadas em distintas regiões do município. Foram incluídas no estudo oito enfermeiros que realizam atendimento pré-natal na Atenção Primária à Saúde. Assim, foram excluídos os profissionais que estavam afastados, de atestado médico, em férias no período da coleta de dados ou que não aceitaram participar.

Foi realizada uma entrevista semiestruturada em profundidade, cujo instrumento foi constituído por dois conjuntos, um referente a dados socioeconômicos dos enfermeiros e outro, referente às questões abertas sobre as condutas utilizadas por estes profissionais durante a consulta de enfermagem, na realização do pré-natal às gestantes com sífilis, bem como, as orientações instituídas e os desafios enfrentados na adesão ao tratamento. Foi realizado um teste piloto para validação das perguntas norteadoras.

A coleta de dados ocorreu em maio de 2021 e foi realizada em dias e horários acordados previamente com os enfermeiros, de maneira individual e em local reservado, sem interrupção. Foi utilizado um dispositivo de gravador de voz para registro das respostas, e um diário de campo.

As entrevistas foram gravadas, posteriormente transcritas na íntegra e em seguida apagadas. A duração média das entrevistas com os participantes foi de 20 minutos, considerando a interação inicial e a entrevista propriamente dita. Ao término das entrevistas o pesquisador perguntou se os entrevistados teriam mais alguma contribuição para o estudo, bem como, se gostariam de fazer alguma alteração no conteúdo.

Os dados foram analisados a partir da técnica de análise temática, Minayo (2014) a saber: pré-análise, com escolha dos documentos analisados e na retomada das hipóteses e dos objetivos iniciais da pesquisa; exploração do material, a partir de uma operação classificatória que visou alcançar o núcleo de compreensão do texto; e tratamento dos resultados obtidos e interpretação, onde as informações obtidas foram interpretadas.

Visando ao anonimato dos enfermeiros e melhor entendimento da análise realizada, o nome dos participantes foi substituído pela letra E, seguida pelo número correspondente à ordem de execução das entrevistas.

Esta pesquisa foi iniciada após aprovação pelo Comitê de Ética em Pesquisa - CAEE: 44357021.7.0000.9727, parecer n. 4.638.519, e após a obtenção do termo de consentimento livre e esclarecido pelos enfermeiros participantes do estudo.

\section{Resultados e Discussão}

Participaram do estudo sete enfermeiras e um enfermeiro das seis unidades de saúde selecionadas. A partir dos dados obtidos por meio da entrevista, foi construído o Quadro 01, no qual constam a caracterização dos enfermeiros participantes.

Quadro 1 - Perfil profissional de enfermeiros de unidades de saúde em um município do Sudoeste do Paraná, 2021

\begin{tabular}{|c|c|c|c|c|c|}
\hline ID & Sexo & Idade & $\begin{array}{c}\text { Tempo de formação } \\
\text { (anos) }\end{array}$ & $\begin{array}{c}\text { Tempo de atuação na unidade } \\
\text { da coleta dos dados }\end{array}$ & $\begin{array}{c}\text { Especialização na área de saúde } \\
\text { pública }\end{array}$ \\
\hline E1 & F & 38 & 13 & 13 anos & Sim \\
\hline E2 & M & 36 & 12 & 02 anos & Sim \\
\hline E3 & F & 31 & 08 & 01 ano e 09 meses & Não \\
\hline E4 & F & 40 & 17 & 01 ano & Não \\
\hline E5 & F & 44 & 21 & 01 ano e 05 meses & Não \\
\hline E6 & F & 33 & 09 & 02 anos & Não \\
\hline E7 & F & 46 & 16 & 06 meses & Não \\
\hline E8 & F & 34 & 10 & 06 meses & \\
\hline
\end{tabular}

Fonte: Autores. 
A média de idade dos profissionais foi de 38 anos (mínimo de 31 e máximo 46 anos). A média de tempo de formação foi de 13 anos (mínimo de 08 e máximo 21 anos). A média de atuação profissional na estratégia saúde da família foi de 02 anos e 09 meses, com variação de 06 meses a 13 anos.

Conforme observado no Quadro 01, a maioria dos entrevistados é do sexo feminino, sendo que as mulheres representam a maior parte dos trabalhadores da área da saúde, ocupando $65 \%$ dos mais de seis milhões de profissionais dos setores público e privado e mais de $80 \%$ na área de enfermagem (Conselho Nacional de Secretarias Municipais de Saúde, 2020).

Após pré-análise das informações obtidas com a leitura, releitura e organização das falas emergiram as categorias: "Primeiro contato com as gestantes no pré-natal e duração das consultas" "Orientação dos enfermeiros sobre a doença" "Estratégias adotadas pelos enfermeiros para adesão ao tratamento da sífilis e comparecimento ao pré-natal" "Desafios enfrentados na adesão ao tratamento e comparecimento às consultas".

\subsection{Primeiro contato com as gestantes no pré-natal e duração das consultas}

O primeiro contato entre o enfermeiro e a gestante ocorre quando a paciente procura o serviço de saúde informando a amenorreia e, assim, são iniciados os exames confirmatórios para gravidez e de rotina. Partindo do pressuposto de que a procura pelo início do pré-natal se dá majoritariamente pela gestante, o acolhimento deve iniciar o mais rápido possível, para que possa ampliar a possibilidade de um atendimento integral e eficaz.

"O primeiro contato é no teste. No dia que ela vem com o teste positivo, ou a gente faz o teste rápido na unidade e ele dá reagente você já inicia o pré-natal, solicita os exames, coleta o preventivo e faz os testes rápidos e preenche a carteirinha, esse é o primeiro contato. A primeira consulta é o retorno com os exames, leva em torno de uma hora e meia a duas horas [...] depende do que você encontra nos exames, as demais consultas que são de retorno, são menores, são vinte minutos, trinta minutos" (E-03).

"[...] começa do momento que elas chegam e dizem: Eu estou suspeita que eu estou gestante. No caso do pré-natal a gente faz o teste rápido de gravidez ou eu solicito o exame de sangue [...] confirmando, eu já início o pré-natal [...] primeira consulta demora aproximadamente uma hora, depois fica mais fluida, vai diminuindo [...]" (E-04).

"A paciente ela vem até a unidade, com amenorreia [...] ou com sintomas de gravidez e a gente já solicita o teste Beta [...] ou pode até fazer um teste rápido [...] então, nós seguimos o protocolo municipal [...] onde o enfermeiro vai fazer o cadastro da gestante, vai preencher a carteirinha da gestante [...] e nesse momento nós fazemos alguns testes rápidos também, que é protocolo [...] Uma hora, no cadastro a gente leva até uma hora e meia” (E-06).

O pré-natal é um momento propício para a realização de atividades educativas, tendo como instrumento o diálogo, o contato e a escuta da opinião da gestante e de seu acompanhante. Essa estratégia de trabalho aproxima a relação entre profissional e gestante, potencializa conhecimentos e esclarece dúvidas (Pohlmann et al., 2016). O acolhimento de qualidade, no primeiro encontro, deve ser marcado por relações de interação, vínculo, confiança, capacidade de escuta, relações horizontais e terapêuticas, entre o enfermeiro e as gestantes. Essas atitudes explicitam o respeito dos profissionais pelas gestantes e contribuem com a assistência pré-natal (Gomes et al., 2019).

A consulta de enfermagem é de suma importância durante todo o pré-natal, uma vez que, por meio da assistência de qualidade é possível identificar intercorrências precocemente, como a sífilis, e monitorar as gestantes que se encontram em situação de risco (Dias et al., 2018). No entanto, o pré-natal deve ser realizado com qualidade pois, estudos realizados no Estado do Amazonas, em 2016 e no Distrito Federal, 2014, apontaram que existe deficiência na qualidade da assistência do pré-natal para gestantes com sífilis, ao considerar que ocorrem muitos diagnósticos tardios e tratamentos inadequados (Lafetá et al., 2016; Soeiro et al., 2014). 
Tem-se que quando o serviço de saúde oferece consultas de enfermagem e busca ativa ocorre diminuição das subnotificações de casos de sífilis em gestantes. Um estudo realizado no Rio Grande do Norte, revelou que municípios com menor disponibilidade de recursos apresentaram maior probabilidade de subnotificações (Carvalho \& Brito, 2014).

\subsection{Orientação dos enfermeiros sobre a doença}

A mulher que for diagnosticada com o teste positivo para sífilis gestacional e não tratada apresenta riscos para o recém-nascido, como a transmissão da infecção, aborto, baixo peso ao nascer e/ou prematuridade. Sendo assim, o tratamento quanto mais próximo ao diagnóstico, apresenta maiores chances do feto não ser contaminado (Brasil, 2019b).

Percebe-se que os entrevistados orientam as gestantes principalmente sobre a forma de transmissão, a importância do tratamento, acompanhamento e os riscos.

"[...] como que é a transmissão, como que acontece a transmissão que pode ser por contato [...]. Oriento sobre a transmissão, as implicações na gestação, a importância do tratamento [...] sobre o acompanhamento [...] e vou esclarecendo se ela tem alguma dúvida [...]” (E-01).

"[...] explica que geralmente é uma doença sexualmente transmissível, que pode não ter sintoma nenhum, que tem tratamento [...]" (E-02).

“[...] explica que é uma doença sexualmente transmissivel, as formas de transmissão, [...] orienta toda a questão do risco para criança" (E-03).

“[...] geralmente explico que é uma doença contagiosa através da relação sexual desprotegida, e geralmente eu tento mais ir para o lado do bebê, o que vai acontecer com o feto, às más formações se ela não fizer o tratamento adequado [...]" (E-08).

O enfermeiro durante a consulta e acompanhamento pré-natal deve manter a gestante informada sobre as infecções sexualmente transmissíveis, principalmente sobre formas de transmissão e prevenção, explicar os benefícios do diagnóstico precoce tanto para a gestante quanto para o bebê e a eficácia de um tratamento de qualidade. É imprescindível que haja um bom acolhimento para fortalecer e criar vínculos entre profissional e usuário (Brasil, 2006).

Estudo realizado no sul do país apontou ainda que, observam-se diagnósticos tardios para sífilis gestacional, sendo que, as complicações tornam-se mais sérias e difíceis de serem amenizadas. Destaca também que, o pré-natal de qualidade é uma importante ferramenta para o diagnóstico, orientação e acompanhamento da gestante na detecção da sífilis gestacional, visando à sua profilaxia precoce, evitando a infecção do recém-nascido (Silva et al., 2019).

As orientações sobre a sífilis no período gestacional são importantes, pois reinfecções foram constatadas pelo fato de não se protegerem com métodos contraceptivos de barreira ou, devido ao tratamento de apenas um dos parceiros (Padovani, Oliveira \& Pelloso, 2016). Outro estudo afirmou que $83 \%$ das mulheres com sífilis não tiveram seus parceiros tratados (Calvacante, Pereira \& Castro, 2017), corroborando outra pesquisa revelou que apenas 22,3\% dos parceiros de mulheres com sífilis gestacional realizam o tratamento (Barbosa et al., 2018). Esses dados apontam que ainda há falhas na comunicação e orientações acerca da sífilis e na sua prevenção e tratamento durante a gestação (Padovani, Oliveira \& Pelloso, 2016).

\subsection{Estratégias adotadas pelos enfermeiros para uma boa adesão ao tratamento da sífilis e comparecimento no pré- natal}

A ação educativa preventiva é uma estratégia importante no controle da sífilis. No âmbito das funções do enfermeiro, a educação em saúde é um fator relevante. O conhecimento técnico-científico do enfermeiro é fundamental e deve ser utilizado 
para compartilhar informações pertinentes relacionadas à manutenção da saúde da gestante e seu bebê (Rodrigues et al., 2009). Nas falas a seguir, relatam-se as estratégias utilizadas:

"[...] vai realizar o teste rápido, principalmente na abertura do pré-natal [...] e se ele der positivo já comunica o médico da equipe, que já trata no dia mesmo do diagnóstico [...] e orienta certinho a periodicidade, que vai ser as próximas doses" (E-02).

"Quando ela tem o diagnóstico e precisa fazer o tratamento, a gente enfatiza, a importância e que principalmente é um tratamento dolorido. A maioria faz três doses, mas isso é critério médico [...] e fala também das consequências, se não fizer o tratamento adequado o que pode ocorrer com a criança" (E-05).

"Quando dá positivo mesmo que seja o teste rápido já inicia o tratamento na hora, depois é solicitado os outros exames de controle" (E-08).

“[...] oportunizar a testagem já na primeira consulta, assegurar que ela já saia da unidade com a primeira dose do tratamento, então o nosso protocolo diante um teste a gestante ela tem que sair com a dose" (E-10).

Nas entrevistas acima, os enfermeiros citaram o teste rápido como o primeiro passo ao iniciar o pré-natal, mesmo sem ter feito os demais exames laboratoriais confirmatórios, para assegurar que elas já saiam da unidade com a primeira dose do tratamento. $\mathrm{O}$ uso dos testes rápidos visa disponibilizar oportunamente o resultado dos exames e o início precoce do tratamento nos casos reagentes de sífilis.

“[...] Vai acompanhando pelo VDRL [...] se a titulação está aumentando, a gente acompanha” (E-04).

"[...] Ela tem que vir até a unidade fazer as medicações, eles dão por três semanas, nós acompanhamos essas aplicações, a gente registra, depois dessas aplicações, um mês depois, começamos a pedir o VDRL para controle e nós continuamos acompanhando [...]” (E-06).

A saúde pública brasileira estabelece vínculos e responsabilidades com os usuários, distribui folders informativos, preservativos, desenvolve intervenções preventivas a fim de evitar a transmissão das infecções sexualmente transmissíveis. Em caso de diagnóstico positivo o cenário deve se caracterizar por um espaço de diálogo, sem julgamentos e com apoio emocional às gestantes.

No momento em que a gestante busca o serviço de atenção primária, para dar início à assistência de pré-natal, a mesma passa a ser responsabilidade do município, sendo que, o pré-natal com qualidade e integral a todas as gestantes seria a maneira mais lógica de se eliminar a sífilis materna e suas consequências (Cabral et al., 2017).

Nesse sentido, entende-se, que a maneira mais concreta de alcançar a prevenção e o controle da sífilis congênita está no compromisso que a Atenção Primária à Saúde oferece às gestantes (Lima et al., 2013).

\subsection{Desafios enfrentados na adesão ao tratamento e comparecimento às consultas}

Alguns enfermeiros expuseram que a falta de conhecimento e poder socioeconômico das gestantes diagnosticadas com sífilis aumenta as chances de haver maiores dificuldades em relação à adesão ao tratamento.

"[...] acredito que talvez a falta de esclarecimento por parte da gestante, às vezes até a questão de entendimento dela, da gravidade da situação. Esses casos são os que mais dificultam o nosso dia a dia, mais a falta de compreensão mesmo por parte delas" (E-01).

"O desafio é principalmente em regiões da cidade que são mais precárias em termos sociais, eu acho que esse é o maior desafio, porque quanto maior o nível social, quanto melhor a estrutura da região a gente percebe que tem mais adesão ao pré-natal [...]" (E-02). 
Quanto menor é o grau de acesso às informações a respeito de doenças, principalmente as sexualmente transmissíveis, mais o indivíduo se torna propenso a desenvolvê-las. Muitas mulheres acabam se reinfectando pela sífilis por não concluírem o tratamento adequadamente. A população deve ser bem informada sobre as formas de transmissão, prevenção e tratamento, principalmente no caso de gestantes em que os riscos envolvem principalmente o concepto (Brasil, 2019b).

Sabe-se os muitos desafios enfrentados para interromper a cadeia de transmissão vertical da sífilis, assim, faz-se necessário priorizar o enfrentamento da sífilis congênita com ações adequadas às necessidades de cada município. Destaca-se que, é imprescindível o diagnóstico e tratamento precoce, tanto da gestante como do parceiro, preenchimento adequado do cartão da gestante, busca ativa das faltosas, construção de um fluxo de notificação em todas as unidades de saúde e capacitação dos profissionais vinculados ao manejo da sífilis adquirida e na gestação (Soares et al., 2020).

Faz-se necessário uma assistência de pré-natal de qualidade, garantindo um diagnóstico precoce e tratamento adequado para evitar transmissão vertical da doença. Um estudo observou dificuldades das gestantes em aprender e memorizar as informações que receberam durante as consultas de pré-natal, sendo que as participantes relataram não terem conhecimento que a infecção poderia ser transmitida ao feto, nem que há a necessidade de se tratar concomitantemente o parceiro (Lima et al., 2013).

\section{Conclusão}

O estudo verificou que durante as consultas de enfermagem, na assistência prestada pelo enfermeiro, para as gestantes com sífilis durante o pré-natal segue as normatizações e protocolos técnicos do Ministério da Saúde, no que tange o cuidado integral na prevenção da sífilis congênita. As práticas utilizadas pelos enfermeiros podem refletir na qualidade da assistência e indicadores de saúde.

É necessário dar ênfase na importância de realizar busca ativa das gestantes para um início precoce do pré-natal, visto como uma ação preventiva para evitar agravos durante todo o período gestacional e ao recém-nascido.

Como fatores limitantes do estudo, considera-se a representação de um único cenário, ressaltando-se a necessidade da realização de novas pesquisas sobre essa temática.

\section{Referências}

Barbosa, D. R. M., Almeida, M. G., Silva, A. O., Araújo, A. A., \& Santos, A. G. (2017). Perfil epidemiológico dos casos de sífilis gestacional. Revista de Enfermagem UFPE online, 11(5), 1867-1874. https://periodicos.ufpe.br/revistas/revistaenfermagem/article/viewFile/23335/18934.

Brasil. Ministério da Saúde. (2006). Manual técnico: pré-natal e puerpério: atenção qualificada e humanizada. https://bvsms.saude.gov.br/bvs/publicacoes/manual_pre_natal_puerperio_3ed.pdf.

Brasil. Ministério da Saúde. (2015). Protocolo clínico e diretrizes terapêuticas para atenção integral às pessoas com infecções sexualmente transmissíveis

Brasil. Ministério da Saúde. (2019a). Boletim epidemiológico: Sífilis 2019. http://www.aids.gov.br/pt-br/pub/2019/boletim-epidemiologico-sifilis-2019.

Brasil. Ministério da Saúde. (2019b). Protocolo clínico e diretrizes terapêuticas para prevenção da transmissão vertical do HIV, sífilis e hepatites virais. http://www.aids.gov.br/pt-br/pub/2015/protocolo-clinico-e-diretrizes-terapeuticas-para-prevencao-da-transmissao-vertical-de-hiv.

Brasil. Ministério da Saúde. (2019c). Sífilis: o que é, sintomas, tratamento e prevenção.

Cabral, B. T. V., Dantas, J. C., Silva, J. A., \& Oliveira, D. A. (2017). Sífilis em gestante e sífilis congênita: um estudo retrospectivo. Revista Ciência Plural, 3(3), 32-44, 22. https://periodicos.ufrn.br/rcp/article/view/13145/9351. doi: https://doi.org/10.21680/2446-7286.2017v3n3ID13145.

Carvalho, I. S. \& Brito, R. S. (2014). Sífilis congênita no Rio Grande do Norte: estudo descritivo do período 2007-2010. Epidemiologia e Serviços de Saúde 23(1), 287-294. http://www.scielo.br/pdf/ress/v23n2/1679-4974-ress-23-02-00287.pdf. doi: 10.5123/S1679-49742014000200010.

Cavalcante, P. A. M., Pereira, R. B. L., \& Castro, J. G. D. (2017). Sífilis gestacional e congênita em Palmas, Tocantins, 2007-2014. Epidemiologia \& Serviços de Saúde, 26(2), 255-264. doi: https://doi.org/10.5123/S1679-49742017000200003.

Conselho Nacional de Secretarias Municipais de Saúde. (2020). Protagonismo feminino na saúde: mulheres são a maioria nos serviços e na gestão do SUS. https://www.conasems.org.br/o-protagonismo-feminino-na-saude-mulheres-sao-a-maioria-nos-servicos-e-na-gestao-do-sus/. 
Dias, E. G., Anjos, G. B., Alves, L., Pereira, S. N., \& Campos, L. N. (2018). Ações do enfermeiro no pré-natal e a importância atribuída pelas gestantes. Revista Sustinere, 6(1), 52-62. https://www.e-publicacoes.uerj.br/index.php/sustinere/article/view/31722. doi: https://doi.org/10.12957/sustinere.2018.31722.

Gomes, C. B. A., Dias, R. S., Silva, W. G. B., Pacheco, M. A. B., Sousa, F. G. M., \& Loyola, C. M. D. (2019). Prenatal nursing consultation: narratives of pregnant women and nurses. Texto \& Contexto-Enfermagem, 28, 1-15. https://www.scielo.br/j/tce/a/3pLDtXNvjLGJWdFFHM3FQbv/?format=pdf\&lang=en. doi: https://doi.org/10.1590/1980-265X-TCE-2017-0544.

Lafetá, K. R. G., Martelli Júnior H., Silveira, M. F., \& Paranaíba, L. M. R. (2016). Sífilis materna e congênita, subnotificação e difícil controle. Revista Brasileira de Epidemiologia, 19(1), 63-74. https://www.scielo.br/j/rbepid/a/dD66wTDCqQrXG3tzt6PqDYx/?format=pdf\&lang=pt. doi: https://doi.org/10.1590/1980-5497201600010006.

Lima, G. C., Dias, I. C. X., Araújo, F. M, Souza, S. B., Sales, D. S., \& Ferreira, A. G. N. (2013). Educação em saúde sobre sífilis com um grupo de gestantes: um relato de experiência de acadêmicas de enfermagem. Sanare: Revista de Políticas Públicas, 12(2), 59-62. https://sanare.emnuvens.com.br/sanare/article/view/384.

Luppi, C. G., Domingues, C. S. B., \& Gomes, S. C. (Orgs.). (2016). Guia de bolso para o manejo da sífilis em gestantes e sífilis congênita. (2a ed.). Secretaria de Estado da Saúde. https://saude.campinas.sp.gov.br/doencas/sifilis/guiadebolsodasifilis_2edicao2016.pdf.

Minayo, M. C. D. S. (2014). O desafio do conhecimento: pesquisa qualitativa em saúde. (14a ed.). Hucitec.

Padovani, C., Oliveira, R. R., \& Pelloso, S. M. (2018). Sífilis na gestação: associação das características maternas e perinatais em região do sul do Brasil. Revista Latino-Americana de Enfermagem, 26, 1-10. https://www.scielo.br/j/rlae/a/KXZGyqSjq4kVMvTL3sFP7zj/?format=pdf\&lang=pt. doi: http://dx.doi.org/10.1590/1518-8345.2305.3019.

Pohlmann, F. C., Kerber, N. P. C., Pelzer, M. T., Dominguez, C. C., Minasi, J. M., \& Carvalho, V. F. (2016). Modelo de assistência pré-natal no extremo sul do país. Texto \& Contexto-Enfermagem, 25(1), 1-8. https://www.scielo.br/j/tce/a/8HrrkWkDG7W6RJP5Sd7gWWS/?lang=pt\&format=pdf. doi: https://doi.org/10.1590/0104-0707201600003680013.

Rodrigues, A. D., Dallanora, C. R., Rosa, J., \& Germani, A. R. M. (2009). Sala de espera: um ambiente para efetivar a educação em saúde. Vivências: Revista Eletrônica de Extensão da URI, 7(5), 101-106, http://www2.reitoria.uri.br/ vivencias/Numero_007/artigos/artigos_vivencias_07/Artigo_13.pdf.

Santos, H. F. L., \& Araujo, M. M. (2016). Políticas de humanização ao pré-natal e parto: uma revisão de literatura. Revista Científica FacMais, 6(2), 54-64. https://revistacientifica.facmais.com.br/wp-content/uploads/2016/07/Artigo-6-POL\%C3\%8DTICAS-DE-HUMANIZA\%C3\%87\%C3\%83O-AO-PR\%C3\%89NATAL-E-PARTO.pdf.

Silva, J. G., Gomes, G. C., Ribeiro, J. P., Jung, B. C., Nörberg, P. K. O., \& Mota, M. S. (2019). Sífilis gestacional: repercussões para a puérpera. Cogitare Enfermagem, 24, 1-11. https://revistas.ufpr.br/cogitare/article/view/65578/pdf. doi: http://dx.doi.org/10.5380/ce.v24i0.65578.

Soares, K. K. S., Prado, T. N., Zandonade, E., Moreira-Silva, S. F., \& Miranda, A. E. (2020). Análise espacial da sífilis em gestantes e sífilis congênita no estado do Espírito Santo, 2011-2018. Epidemiologia e Serviços de Saúde, 29(1), 1-11. doi: https://doi.org/10.5123/S1679-49742020000100018.

Soeiro, C. M. O., Miranda, A. E., Saraceni, V., Santos, M. C., Talhari, S., \& Ferreira, L. C. L. (2014). Syphilis in pregnancy and congenital syphilis in Amazonas State, Brazil: an evaluation using database linkage. Cadernos de Saúde Pública, 30(4), 715-723. https://www.scielo.br/j/csp/a/D8sckxWDvFgJy8HKGw78ZYn/?format=pdf\&lang=en. doi: 10.1590/0102-311x00156312.

Suto, C. S. S., Silva, D. L., Almeida, E. S., Costa, L. E. L., \& Evangelista, R. J. (2016). Assistência pré-natal a gestante com diagnóstico de sífilis. Revista de Enfermagem e Atenção à Saúde, 5(2), 18-33. http://seer.uftm.edu.br/revistaeletronica/index.php/enfer/article/view/1544. doi: https://doi.org/10.18554/reas.v5i2.1544. 